\title{
Anomalous Hall effect scaling in ferromagnetic thin films
}

\author{
Vahram L. Grigoryan, ${ }^{1,2}$ Jiang Xiao (萧江), ${ }^{3,4,5,{ }^{*}}$ Xuhui Wang, ${ }^{6, \dagger}$ and Ke Xia ${ }^{1,2,7, \ddagger}$ \\ ${ }^{1}$ The Center for Advanced Quantum Studies and Department of Physics, Beijing Normal University, Beijing 100875, China \\ ${ }^{2}$ Beijing Normal University, Zhuhai 519087, Guangdong, China \\ ${ }^{3}$ Department of Physics and State Key Laboratory of Surface Physics, Fudan University, Shanghai 200433, China \\ ${ }^{4}$ Collaborative Innovation Center of Advanced Microstructures, Nanjing 210093, China \\ ${ }^{5}$ Institute for Nanoelectronics Devices and Quantum Computing, Fudan University, Shanghai 200433, China \\ ${ }^{6}$ King Abdullah University of Science and Technology (KAUST), Physical Science and Engineering Division, \\ Thuwal 23955-6900, Saudi Arabia \\ ${ }^{7}$ Synergetic Innovation Center for Quantum Effects and Applications (SICQEA), Hunan Normal University, Changsha 410081, China
}

(Received 5 July 2016; revised manuscript received 31 August 2017; published 23 October 2017)

\begin{abstract}
We propose a scaling law for anomalous Hall effect in ferromagnetic thin films. Our approach distinguishes multiple scattering sources, namely, bulk impurity, phonon for Hall resistivity, and most importantly the rough surface contribution to longitudinal resistivity. In stark contrast to earlier laws that rely on temperature- and thickness-dependent fitting coefficients, this scaling law fits the recent experimental data excellently with constant parameters that are independent of temperature and film thickness, strongly indicating that this law captures the underlying physical processes. Based on a few data points, this scaling law can even fit all experimental data in full temperature and thickness range. We apply this law to interpret the experimental data for $\mathrm{Fe}, \mathrm{Co}$, and $\mathrm{Ni}$ and conclude that (i) the phonon-induced skew scattering is unimportant as expected; (ii) contribution from the impurity-induced skew scattering is negative; (iii) the intrinsic (extrinsic) mechanism dominates in $\mathrm{Fe}(\mathrm{Co})$, and both the extrinsic and intrinsic contributions are important in Ni.
\end{abstract}

DOI: 10.1103/PhysRevB.96.144426

\section{INTRODUCTION}

In normal metals, the Hall effect, a charge current transverse to both the applied magnetic and electric field, is known to be driven by the Lorentz force. In ferromagnetic metals, yet a very similar effect, known as the anomalous Hall effect (AHE), is present without magnetic field [1,2]. The real physical mechanisms behind AHE are under debate for decades. Spin-orbit coupling (SOC), believed to be responsible for AHE, brings two types of mechanisms, i.e., the intrinsic and the extrinsic one [2]. The intrinsic mechanism, arising from the SOC intrinsic to the band structure, was first proposed by Karplus and Luttinger [3] and later reformulated in terms of Berry's phase [4]. The extrinsic mechanism, due to scatterings with impurities carrying SOC, gives rise to two contributions known as skew scattering [5,6] and side jump [7]. Asymmetric scattering by impurities leads to the skew scattering, while the side jump originates from that electrons with opposite spins are deflected to opposite directions when scattered by an impurity.

Progress made in the past indicates that the intrinsic mechanism shall dominate in AHE [8]. The theories suggest that the Hall resistivity $\left(\rho_{\mathrm{AH}}\right)$ and longitudinal resistivity $\rho$ have the following scaling relations: $\rho_{\mathrm{AH}}^{\mathrm{INT}}, \rho_{\mathrm{AH}}^{\mathrm{SJ}} \propto \rho^{2}$ for both intrinsic [3] and extrinsic side-jump mechanisms [7], while $\rho_{\mathrm{AH}}^{\mathrm{SS}} \propto \rho$ is for the extrinsic skew-scattering mechanism [5,6]. Such a scaling law helps to outline the underlying picture. However, this simple relation $\rho_{\mathrm{AH}} \sim a \rho+b \rho^{2}$ often breaks down when a comparison with experiments is made.

\footnotetext{
*Corresponding author: xiaojiang@ fudan.edu.cn

${ }^{\dagger}$ Present address: Kwantum Links, Benoordenhoutseweg 23, 2596 BA, The Hague, The Netherlands.

${ }^{\ddagger}$ Corresponding author: kexia@bnu.edu.cn
}

To resolve this issue [9], Jin's group in Fudan University, by systematically varying the film thickness and temperature, has measured both the longitudinal and anomalous Hall resistivities in $\mathrm{Fe}, \mathrm{Co}$, and $\mathrm{Ni}$ thin films [10-13]. This experimental paradigm does not affect the sample's band structure and leaves the intrinsic contribution untouched. Hou et. al. [13] argued that, while most theories only assume a single type of scatterer, the real complexity in the experimental data arises from the fact that there are more than one type of impurity scatterers. By assuming multiple scatterers, Hou et. al. proposed a scaling law [13]

$$
\rho_{\mathrm{AH}}=c \rho_{x x}^{2}+\sum_{i} c_{i} \rho_{i} \rho_{\mathrm{xx}}+\sum_{i j} c_{i j} \rho_{i} \rho_{j}+\sum_{i \in S} \alpha_{i} \rho_{i},
$$

where $\rho_{x x}=\sum_{i} \rho_{i}$ is the overall longitudinal resistivity, $\rho_{i}$ the partial longitudinal resistivity from $i$ th scattering source, and $S$ represents the static scattering sources that remain at zero temperature. In addition, $c, c_{i}, c_{i j}$, and $\alpha_{i}$ are constant coefficients. We believe this relation points to a plausible direction for resolving the AHE scaling issue. Unfortunately, this scaling law not only carries extreme complexity, but also is very difficult to test since the partial resistivities are usually not directly measurable.

In this paper, we develop a theory based on semiclassical Boltzmann equation (SBE) to describe the AHE in ferromagnetic thin films. From a pure quantum mechanical point of view, SBE is simple and intuitive, although its applicability is not universal. In the pursuit of full quantum mechanical version of, for example, the conductivity, one often turns to linear response (Kubo formalism). However, a comparative study on these two techniques has shown [14] that SBE does provide a simple semiclassical interpretation of the Kubo formalism. When it comes to calculate the 
Hall current, these two approaches converge to the same expressions. Thus, using a method based on SBE, taking into account the finite thickness and scattering by the bulk impurities, phonons, and surface roughness, we calculate the longitudinal resistivity $\rho$, transverse resistivity $\rho_{\mathrm{AH}}$, and their scaling relation. We find excellent agreement between our theory and the experimental data on $\mathrm{Fe}, \mathrm{Co}$, and $\mathrm{Ni}$ from Jin's group [10-13]. For $\rho$, the film surface roughness is the only fitting parameter; for $\rho_{\mathrm{AH}}$, there are only four constant coefficients accounting the impurity-induced side jump and skew scattering, phonon-induced side jump, and intrinsic contributions. We are thus able to identify the proportion of each contribution. We conclude that the intrinsic (extrinsic) effect dominates in $\mathrm{Fe}(\mathrm{Co})$. Yet in $\mathrm{Ni}$, the intrinsic and extrinsic effects are both important and in competition.

We proceed with our discourse in the following four sections. The theoretical model in Sec. II introduces the Hamiltonian of the system and the scattering sources; calculations of relaxation times due to different scattering sources are derived in Sec. III, derivation of longitudinal and transverse resistivities are presented in Sec. IV. Finally, we discuss the results and summarize in $\mathrm{Sec} . \mathrm{V}$.

\section{MODEL}

The Hamiltonian for a ferromagnetic thin film with a magnetization $\mathbf{m}$ and a constant thickness $d$ is

$$
\hat{H}_{0}=\frac{\hat{\mathbf{p}}^{2}}{2 m}+V_{d}(z)+V_{\mathrm{sd}},
$$

where $m$ is the electron mass and $\hat{\mathbf{p}}=-i \hbar \nabla$ the momentum operator. The confining potential is $V_{d}(z)=U_{0} \Theta(z-d / 2)+$ $U_{0} \Theta(-z-d / 2)$ with $U_{0}$ the potential height and $\Theta$ the Heaviside unit step function. $V_{\mathrm{sd}}=-J_{\mathrm{sd}} \hat{\boldsymbol{\sigma}} \cdot \mathbf{m}$ is the exchange energy between the itinerant $s$ and the local $d$ electrons. $J_{\text {sd }}$ is $s-d$ coupling constant and the Pauli matrices are $\hat{\boldsymbol{\sigma}}=$ $\left(\hat{\sigma}_{x}, \hat{\sigma}_{y}, \hat{\sigma}_{z}\right)$. The eigensolutions of this unperturbed Hamiltonian $\hat{H}_{0}$ are

$$
\begin{aligned}
E_{n \mathbf{q} s} & =E_{n \mathbf{q} s}-s J_{\mathrm{sd}}, \\
|\alpha\rangle & =|n \mathbf{q} s\rangle=\sqrt{\frac{2}{A d}} \sin \left(k_{n} z\right) e^{i \mathbf{q} \cdot \boldsymbol{\rho}}|s\rangle,
\end{aligned}
$$

where $E_{n \mathbf{q}_{s}}=\hbar^{2}\left(k_{n}^{2}+\mathbf{q}_{s}^{2}\right) / 2 m$ is the kinetic energy with $k_{n}=$ $\pi n / d$ denoting different transverse conducting channels. $\rho=$ $(x, y)$ and $\mathbf{q}_{s}=\left(q_{x, s}, q_{y, s}\right)$ are the in-plane coordinate and wave vector, respectively. The area of the film is $A$. Here, $|s\rangle=| \pm\rangle$ is eigenstate of $\hat{\boldsymbol{\sigma}} \cdot \mathbf{m}|s\rangle=s|s\rangle$.

Now, we focus on scattering mechanisms treated as perturbation. First, we assume a nonmagnetic pointlike bulk impurity scatterer with potential $V_{\mathrm{I}}(\mathbf{r})=V_{\mathrm{imp}} \sum_{i} \delta\left(\mathbf{r}-\mathbf{r}_{i}\right)$ distributed homogeneously in the film. We assume an impurity density $n_{i}$. Position of impurity $i$ is given by $\mathbf{r}_{i}=\left(\boldsymbol{\rho}_{i}, z_{i}\right)$. Next, we consider electron-phonon interaction with effective potential $V_{\mathrm{P}}(\mathbf{r})=V_{\mathrm{p}} \sum_{j} \delta\left(\mathbf{r}-\mathbf{R}_{j}\right)$, with lattice coordinate $\mathbf{R}_{j}$. Furthermore, the gradient of the impurity (phonon) potential $V_{\mathrm{I}, \mathrm{P}}$ gives rise to a spin-orbit (spin-phonon $[15,16])$ coupling

$$
V_{\mathrm{I}, \mathrm{P}}^{\mathrm{SO}}(\mathbf{r})=\frac{\eta}{\hbar}\left[\hat{\boldsymbol{\sigma}} \times \nabla V_{\mathrm{I}, \mathrm{P}}(\mathbf{r})\right] \cdot \hat{\mathbf{p}},
$$

where $\eta=\mu_{B} \hbar /($ mce $)$ is the coupling constant and $\mu_{B}=e \hbar /(2 m c)$ is Bohr magneton. In addition, we consider a rough surface described by a fluctuating position-dependent thickness $d(\rho)$ with an average $\langle d(\rho)\rangle=d$. The surface roughness is converted into an effective scattering potential [17-19]

$$
V_{\mathrm{R}}(\mathbf{r})=\lambda_{\rho}\left[2 V_{d}(\mathbf{r})+z \partial_{z} V_{d}(\mathbf{r})\right]
$$

by a dilation operator that transforms a varying thickness into a constant one [17]. Here, $\lambda_{\rho}=\ln [d / d(\rho)]$ is the small deviation of the thickness from $d$. We treat it simply as a "white-noise" surface profile, i.e., the surface roughness is uncorrelated and characterized by the dimensionless parameter $\Lambda \sim(\delta / d)^{2}$ with a variance $\delta^{2}$. This leads to a correlation $\left\langle\lambda_{\rho} \lambda_{\rho^{\prime}}\right\rangle=\Lambda a_{0}^{2} \delta\left(\rho-\rho^{\prime}\right)$, given $a_{0}=\pi / k_{\mathrm{F}}$. Accounting all contributions above $U=V_{\mathrm{I}}+V_{\mathrm{P}}+V_{\mathrm{R}}+V_{\mathrm{I}}^{\mathrm{SO}}+V_{\mathrm{P}}^{\mathrm{SO}}$, the total Hamiltonian $\hat{H}=\hat{H}_{0}+U$.

We proceed with Lippmann-Schwinger formalism to calculate the transition probability [20]

$$
P_{n \mathbf{q} s}^{n^{\prime} \mathbf{q}^{\prime} s^{\prime}}=\frac{2 \pi}{\hbar}\left|\left\langle\left\langle n^{\prime} \mathbf{q}^{\prime} s^{\prime}|\hat{T}| n \mathbf{q} s\right\rangle\right\rangle_{\mathrm{en}}\right|^{2} \delta\left(E_{n \mathbf{q} s}-E_{n^{\prime} \mathbf{q}^{\prime} s^{\prime}}\right),
$$

where $\hat{T}=U+U(E-\hat{H})^{-1} U$

\section{RELAXATION TIMES}

For each conduction channel at Fermi energy $E_{\mathrm{F}}$, the relaxation rate is obtained from the symmetric part of the transition probability $[19,21]$

$$
\frac{1}{\tau_{n}^{s}}=\frac{1}{\tau_{n}}\left(1-s \frac{J_{\mathrm{sd}}}{2 E_{\mathrm{F}}}\right) \quad \text { with } \quad \frac{1}{\tau_{n}}=\frac{1}{\tau_{0}}+\frac{1}{\tau_{n}^{\mathrm{R}}},
$$

where $\tau_{n}^{s}\left(\tau_{n}\right)$ is the spin-dependent (-independent) relaxation time for channel $n, \tau_{0}^{-1}=\tau_{\mathrm{I}}^{-1}+\tau_{\mathrm{P}}^{-1}$ is the bulk relaxation rate subscribing to both the impurity- and phonon-induced relaxations. The temperature dependence of $\tau_{0}^{-1}$ can be approximately described by the Block-Grüneisen (BG) theory, i.e., $\tau_{0}^{-1}=a+b T^{c}$ [22-24], and the channel (n)-dependent roughness-induced relaxation rate is $[17,19]$

$$
\frac{1}{\tau_{n}^{\mathrm{R}}}=2 \frac{E_{\mathrm{F}}}{\hbar} \frac{n^{2}}{3 n_{c}^{3}}\left(\frac{\delta}{a_{0}}\right)^{2}
$$

with $n_{c}=\left\lfloor k_{\mathrm{F}} d / \pi\right\rfloor$ the total number of transverse channels and $n \leqslant n_{c}$.

\section{LONGITUDINAL AND ANOMALOUS HALL RESISTIVITIES}

\section{A. Longitudinal resistivity}

Similar to the calculation of the longitudinal and transverse spin Hall conductivities in normal metals [19,20], we calculate them in a ferromagnetic thin film. Following Takahashi and Maekawa [21] in the presence of impurities, the velocity operator

$$
\hat{\mathbf{v}}=\frac{i}{\hbar}[\hat{H}, \mathbf{r}]=\frac{\hat{\mathbf{p}}}{m}+\sum_{j=I, P} \frac{\eta_{j}}{\hbar}\left[\hat{\boldsymbol{\sigma}} \times \nabla V_{j}(r)\right],
$$

where the second term contains the anomalous velocity due to impurity- and phonon-induced SOC and $\eta_{\mathrm{I}, \mathrm{P}}$ are 
the corresponding SOC constants. The side-jump effect is embedded in the expectation value of the anomalous velocity. To calculate the velocity, the Lippmann-Schwinger equation is used to construct a scattering state $\left|\alpha^{+}\right\rangle$which describes the final state as an incoming state $|\alpha\rangle$ undergoing scattering events by the nonmagnetic impurity potential $V_{\mathrm{I}}(\mathbf{r})$ and phonon potential $V_{\mathrm{P}}(\mathbf{r})$. Due to the potentials, the scattered state $\left|\alpha^{+}\right\rangle$ by the Born approximation is

$$
\left|\alpha^{+}\right\rangle=|\alpha\rangle+\sum_{\mathbf{q}^{\prime} n^{\prime}} \frac{V_{\mathrm{I}}^{\mathbf{q}^{\prime} s ; \mathbf{q} s}+V_{\mathrm{P}}^{\mathbf{q}^{\prime} s ; \mathbf{q} s}}{E_{n q}-E_{n^{\prime} q^{\prime}}+i \delta},
$$

where $V_{\mathrm{I}, \mathrm{P}}^{\mathbf{q}^{\prime} ; \mathbf{q} s}$ are the matrix elements of the potentials. The charge current density can be calculated from

$$
\mathbf{J}=\frac{e}{v} \sum_{s, \mathbf{q}, n} f_{\mathbf{q}, n, s} \mathbf{v}_{s c}
$$

where $v$ is the volume and $\mathbf{v}_{s c}=\left\langle\alpha^{+}|\hat{\mathbf{v}}| \alpha^{+}\right\rangle$is the expectation value for velocity calculated using scattered states. $f_{\mathbf{q}, n, s}=$ $f_{\mathbf{q}, n, s}^{(0)}+f_{\mathbf{q}, n, s}^{(1)}+f_{\mathbf{q}, n, s}^{(2)}$ is the distribution function with $f_{\mathbf{q}, n, s}^{(0)}=$ $\Theta\left(E_{\mathbf{q} n s}-E_{F}\right)$ being the equilibrium Fermi-Dirac distribution

$$
f_{\mathbf{q}, n, s}^{(1)}=\frac{e \hbar}{m} \tau_{n}^{s} \boldsymbol{\varepsilon} \cdot \mathbf{q}_{s} \delta\left(E_{\mathbf{q} n s}-E_{F}\right)
$$

and

$$
f_{\mathbf{q}, n, s}^{(2)}=\frac{e \hbar}{m} \tau_{n}^{s} \sum_{\mathbf{q}^{\prime} n^{\prime} s^{\prime}} \tau_{n^{\prime}}^{s^{\prime}} P_{n \mathbf{q} s}^{n^{\prime} \mathbf{q}^{\prime} s^{\prime}(2)} \boldsymbol{\varepsilon} \cdot \mathbf{q}_{s} \delta\left(E_{\mathbf{q}^{\prime} n^{\prime} s^{\prime}}-E_{F}\right)
$$

are the first- and second-order corrections due to electric field $\boldsymbol{\varepsilon}$, where $P_{n \mathbf{q} s}^{n^{\prime} s^{\prime}(2)}$ is the antisymmetric part of the transition probability (6).

The in-plane longitudinal conductivity is given by the term in Eq. (11) that is the product of the normal velocity and $f^{(1)}$ [17-20]:

$$
\rho^{-1}=\sigma(T, d, \delta)=\frac{3 \sigma_{0}}{2 n_{c}} \sum_{n}^{n_{c}} \frac{\tau_{n}}{\tau_{0}}\left(1-\frac{n^{2}}{n_{c}^{2}}\right),
$$

where we used the relaxation rates in Eqs. (7) and (8), and $\sigma_{0}=n_{e} e^{2} \tau_{0} / m$ is the temperature-dependent bulk Drude conductivity with an electron density $n_{e}=k_{\mathrm{F}}^{3} / 3 \pi^{2}$.

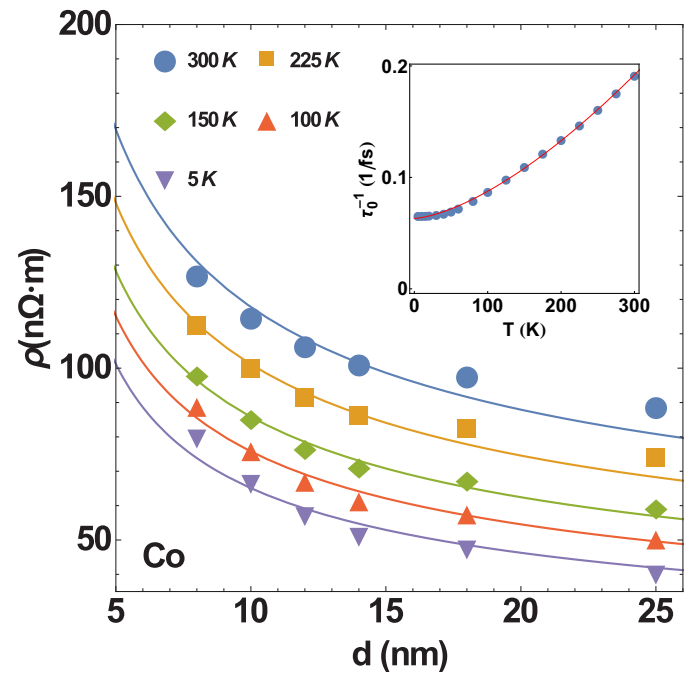

FIG. 1. The thickness dependence of the longitudinal resistivity at various temperatures. Curves at different temperatures have different $\tau_{0}$, which is plotted in the inset and can be well described by the BG formula (the red curve). The points are the experimental data for $\mathrm{Co}$ from Ref. [11], the curves are plotted from Eq. (14) with $\delta \approx 5.7 a_{0}$.

We test our longitudinal resistivity Eq. (14) using the experimental data obtained by Jin's group in $\mathrm{Fe}, \mathrm{Co}$, and $\mathrm{Ni}$ [11-13], for which the film thickness and temperature are independently tuned. The only two free parameters in Eq. (14) are the film surface roughness $\delta$ and (temperature-dependent) bulk relaxation time $\tau_{0}(T)$. We assume that $\delta$ is the same for all thicknesses, as the growth condition remains the same for all films. The relaxation time $\tau_{0}$ depends on temperature as it includes the phonon-induced relaxation. Therefore, the data obtained at the same temperature share the same $\tau_{0}$. The fitting of the thickness dependence of the longitudinal resistivity for Co using Eq. (14) is shown in Fig. 1. All fitting curves share the same roughness $\delta / a_{0}=5.7$. The inset shows the temperature dependence of the fitted values for $\tau_{0}$, which reflects the temperature dependence of the bulk (impurity and phonon-induced) relaxation time. The saturated value at low temperatures gives the residual relaxation rate (residual (a)

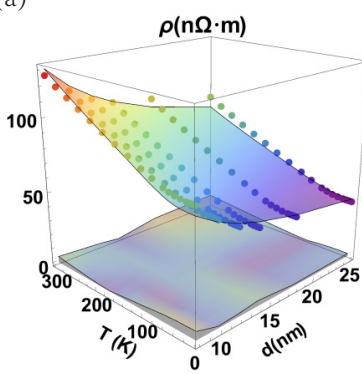

(b)

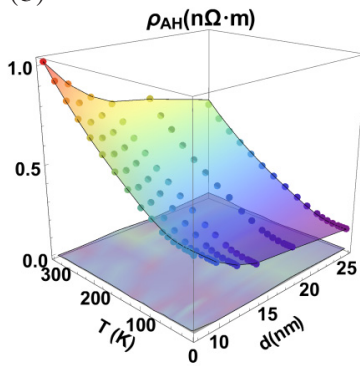

(c)

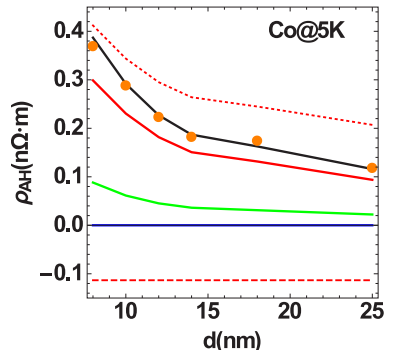

(d)

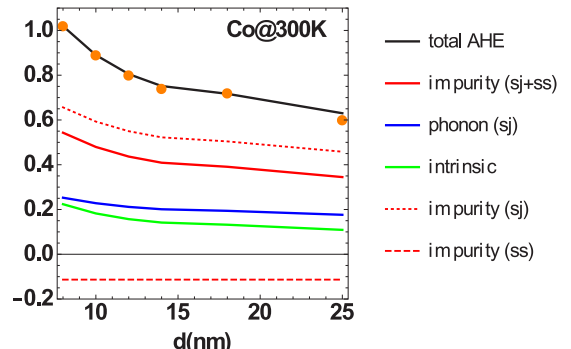

FIG. 2. (a) The thickness and temperature dependence of the longitudinal resistivity. The surface is plotted from Eq. (14). (b) The thickness and temperature dependence of the anomalous Hall resistivity. The surface is plotted from Eq. (18) with $\alpha_{\mathrm{I}}, \alpha_{\mathrm{P}}, \gamma$ as fitting parameters, whose values are listed in Table I. The points are the experimental data for Co from Ref. [11]. The shaded area at the bottom indicates the absolute difference between the experiment and theory. (c), (d) Thickness dependence of extrinsic and intrinsic contributions at low and high temperatures, respectively. The dotted (dashed) red lines are the side-jump (skew-scattering) contribution due to impurity scattering, and their sum is plotted as the solid red line. The blue solid line is the side-jump contribution due to phonon scattering. The solid green line is the intrinsic contribution. 

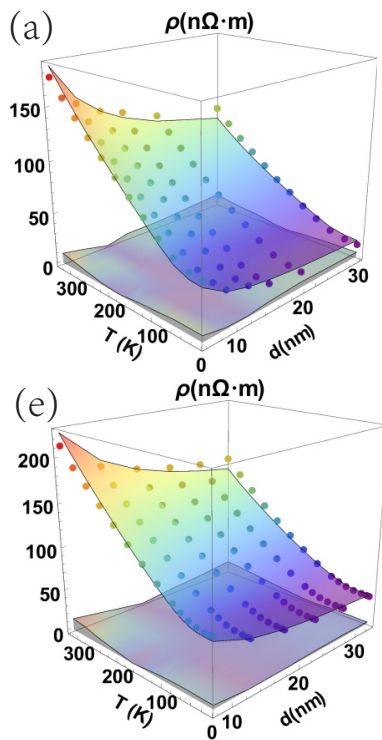
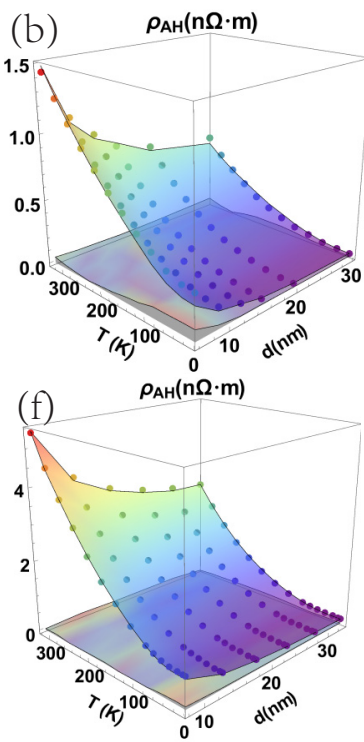
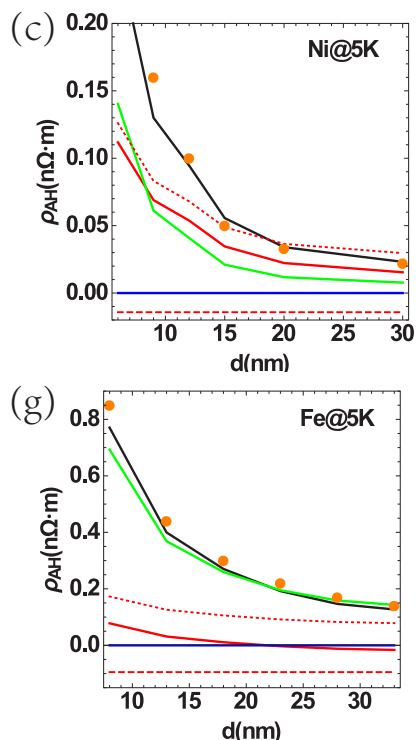
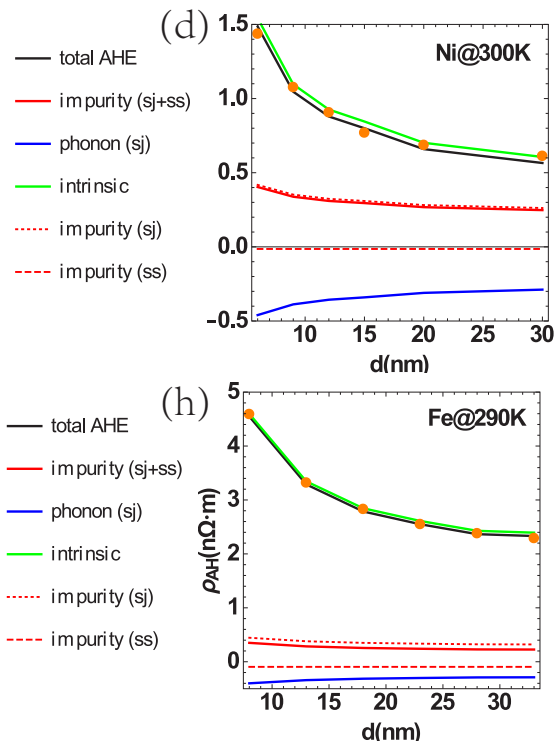

FIG. 3. (Top row) The same as in Fig. 2 for Ni. The points are the experimental data from Ref. [12]. (Bottom row) The same as in Fig. 2 for Fe. The points are the experimental data from Ref. [13].

longitudinal resistivity). The full comparison of Eq. (14) with experiment for Co is shown in Fig. 2(a) (see Fig. 3 for similar plots for $\mathrm{Ni}$ and $\mathrm{Fe}$ ). The increase in the resistivity with decreasing film thickness is due to an enhanced scattering from the surface roughness $[17,19]$.

\section{B. Anomalous Hall resistivity}

We now consider the transverse anomalous Hall conductivity in a ferromagnetic thin film with a magnetization perpendicular to the film plane. Several mechanisms contribute, including the intrinsic contribution from the band structure [3] and the extrinsic contributions, i.e., side jump and skew scattering, originating from the spin-orbit coupling by the impurities and phonons. The intrinsic contribution has been well studied and is assumed to be independent of the sample geometry. The intrinsic anomalous Hall resistivity scales quadratically with the longitudinal resistivity: $\rho_{\mathrm{AH}}^{\mathrm{INT}}=\gamma \rho^{2}[2]$. In terms of conductivities, it is $\sigma_{\mathrm{AH}}^{\mathrm{INT}}=\gamma$. Yet, the extrinsic contributions depend on the scattering processes.

In Eq. (11), the term proportional to the product of anomalous velocities in Eq. (9) and the first-order correction of the distribution function $f^{(1)}$ lead to transverse side-jump current or side-jump conductivity (see Appendix A for details)

$$
\sigma_{\mathrm{AH}}^{\mathrm{SJ}}=\alpha_{\mathrm{I}} \frac{\sigma}{\sigma_{\mathrm{I}}}+\alpha_{\mathrm{P}} \frac{\sigma}{\sigma_{\mathrm{P}}} \quad \text { with } \quad \alpha_{\mathrm{I}, \mathrm{P}}=\eta_{\mathrm{I}, \mathrm{P}} \frac{n_{e} e^{2}}{2 \hbar} \frac{J_{\mathrm{sd}}}{E_{\mathrm{F}}},
$$

where $\alpha_{\mathrm{I}, \mathrm{P}}$ are side-jump coefficients due to impurity/phonon scatterings, and $\sigma_{\mathrm{I}, \mathrm{P}}=n_{e} e^{2} \tau_{\mathrm{I}, \mathrm{P}} / m$ is the bulk Drude conductivity due to impurity/phonon relaxation alone. The skew-scattering contribution can be calculated from Eq. (11) where now we consider the terms proportional to normal velocity and second-order correction of the distribution function $f^{(2)} \propto P_{n \mathbf{q}^{s}}^{n^{\prime} s^{\prime} s^{\prime}(2)}$ (see Appendix B for details):

$$
\begin{aligned}
\sigma_{\mathrm{AH}}^{\mathrm{SS}} & =\beta_{\mathrm{I}} \frac{\sigma^{2}}{\sigma_{\mathrm{I}}}+\beta_{\mathrm{P}} \frac{\sigma^{2}}{\sigma_{\mathrm{P}}} \quad \text { with } \\
\beta_{\mathrm{I}} & =-\eta_{\mathrm{I}} \frac{\pi n_{e} m V_{\mathrm{imp}}}{2 \hbar^{2}} \frac{J_{\mathrm{sd}}}{E_{\mathrm{F}}} \text { and } \\
\beta_{\mathrm{P}} & =-\eta_{\mathrm{p}} \frac{\pi n_{e} n_{i} m V_{\mathrm{imp}}}{2 \hbar^{2}} \frac{J_{\mathrm{sd}}}{E_{\mathrm{F}}},
\end{aligned}
$$

where $\beta_{\mathrm{I}}$ is the skew-scattering coefficient due to impurity scatterings arising from the asymmetric part of the transition probability (6) related to term $\left\langle\left\langle V_{\mathrm{I}}^{2} V_{\mathrm{I}}^{\mathrm{SO}}\right\rangle\right\rangle$, and $\beta_{\mathrm{P}}$ is the skew-scattering contribution from the crossed scattering by phonon and impurity related to term $\left\langle\left\langle V_{\mathrm{I}} V_{\mathrm{P}} V_{\mathrm{P}}^{\mathrm{SO}}\right\rangle\right\rangle[28]$. The skew-scattering terms in $\left\langle\left\langle U^{3}\right\rangle\right\rangle$ that are odd in $V_{R, P}$ vanish after ensemble average due to the fluctuating sign of $V_{R, P}$ $[28,29]$. The overall anomalous Hall conductivity is the sum of all contributions

$$
\sigma_{\mathrm{AH}}=\alpha_{\mathrm{I}} \frac{\sigma}{\sigma_{\mathrm{I}}}+\beta_{\mathrm{I}} \frac{\sigma^{2}}{\sigma_{\mathrm{I}}}+\alpha_{\mathrm{P}} \frac{\sigma}{\sigma_{\mathrm{P}}}+\beta_{\mathrm{P}} \frac{\sigma^{2}}{\sigma_{\mathrm{P}}}+\gamma
$$

and when expressed in terms of resistivity,

$$
\rho_{\mathrm{AH}}=\frac{\sigma_{\mathrm{AH}}}{\sigma^{2}}=\alpha_{\mathrm{I}} \rho_{\mathrm{I}} \rho+\beta_{\mathrm{I}} \rho_{\mathrm{I}}+\alpha_{\mathrm{P}} \rho_{\mathrm{P}} \rho+\beta_{\mathrm{P}} \rho_{\mathrm{P}}+\gamma \rho^{2} .
$$

TABLE I. Fitted values of the coefficients and surface roughness of thin films.

\begin{tabular}{lccccccc}
\hline \hline Material & $k_{\mathrm{F}}(1 / \mathrm{A})$ & $\delta / a_{0}$ & $\alpha_{\mathrm{I}}(1 / \mathrm{m} \Omega \cdot \mathrm{m})$ & $\beta_{\mathrm{I}}\left(10^{-3}\right)$ & $\alpha_{\mathrm{P}}(1 / \mathrm{m} \Omega \cdot \mathrm{m})$ & $\beta_{\mathrm{P}}$ & $\gamma(1 / \mathrm{m} \Omega \cdot \mathrm{m})$ \\
\hline $\mathrm{Fe}[13]$ & $1.71[25]$ & 5.2 & +184 & -7.6 & -29 & 0 & +123 \\
$\mathrm{Co}[11]$ & $1.78[26]$ & 5.7 & +423 & -9.3 & +85 & 0 & +14 \\
$\mathrm{Ni}[12]$ & $1.54[26,27]$ & 4.5 & +728 & -4.4 & -47 & 0 & +50 \\
\hline \hline
\end{tabular}


In the above $\rho(T, d, \delta)$ is the longitudinal resistivity of the thin film and $\rho_{\mathrm{I}, \mathrm{P}}=\sigma_{\mathrm{I}, \mathrm{P}}^{-1}$ is the bulk limit of the Drude resistivity with only the impurity/phonon relaxation.

Our scaling law in Eq. (18) is in line with the general scaling relation [13] in Eq. (1), which includes all possible combinations of different scatterers up to the second order. However, the extreme generality of Eq. (1) also makes it impossible to be applied meaningfully to any experiments. Comparison of our scaling law with a more practical one [Eq. (3) in Ref. [13]], where the authors specified scattering sources (impurities and phonons), demonstrates the importance of our law. Inclusion of scattering by surface roughness in longitudinal resistivity captures the thickness dependence of both longitudinal and transverse resistivities, leading to thickness-independent parameters in scaling law. In contrast, the fitting parameters in previous relations [13] depend on thickness, which increases the number of fitting parameters dramatically. In our Eq. (18) three types of scatterers (impurity, phonon, and surface roughness for longitudinal and impurity/phonons for transverse conductivities) are included in parameters $\alpha_{\mathrm{I}, \mathrm{P}}, \beta_{\mathrm{I}, \mathrm{P}}, \gamma$, which are material constants and are independent of the film thickness/roughness and temperature. As the central result of this paper, Eq. (18) provides the needed scaling law for the anomalous Hall effect in a ferromagnetic thin film with merely five constant coefficients.

The law (18) is tested against the experimental data from Jin's group. We first extract the impurity-induced skewscattering parameter $\beta_{\mathrm{I}}$ in Eq. (18) by fitting the experimental data with varying thickness but at low temperatures $\left(\rho_{\mathrm{P}} \simeq 0\right)$. In this case, Eq. (18) reduces to a quadratic function of $\rho$, and the interception at $\rho=0$ is $\beta_{\mathrm{I}} \rho_{\mathrm{I}}$. The $\beta_{\mathrm{P}}$ term gives significant contribution in alloys [28], it is typically small in pure metals studied in this paper, therefore, we set $\beta_{\mathrm{P}}=0$. With $\beta_{\mathrm{I}, \mathrm{P}}$ fixed, the full experimental data are fitted using Eq. (18) with $\alpha_{\mathrm{I}, \mathrm{P}}$ and $\gamma$ as fitting parameters. The fitted values for $\alpha_{\mathrm{I}, \mathrm{P}}, \beta_{\mathrm{I}, \mathrm{P}}$, and $\gamma$ are listed in Table I. The comparison of the fitted $\rho_{\mathrm{AH}}$ in Eq. (18) (surface) with the experimental data (points) for Co is shown in Fig. 2(b) (see Fig. 3 for similar plots for Ni and Fe).
The thickness dependence of the various AHE contributions in Co is shown in Figs. 2(c) and 2(d) at low and room temperatures, from which we see that extrinsic contributions dominate in Co, regardless the temperature. Figure 4(b) plots the ratio between the intrinsic contribution and the total $\mathrm{AHE}$ $r_{\mathrm{INT}}=\rho_{\mathrm{AH}}^{\mathrm{INT}} / \rho_{\mathrm{AH}}$, which is about $20 \%$ of the total AHE for Co in the full temperature and thickness range. This means that the extrinsic mechanisms dominate in Co. On the other hand, the intrinsic effect dominates in Fe with $r_{\text {INT }}>90 \%$ in the full range in $\mathrm{Fe}$. In $\mathrm{Ni}$, the intrinsic (extrinsic) effect dominates at high (low) temperatures. One should notice that our conclusions concerning the intrinsic contribution in $\mathrm{Ni}$ differ from the original experimental work in Ref. [12], where the authors claim that the intrinsic contribution dominates in the whole temperature range. The conclusion on which mechanism is dominating relies highly on the scaling law used for interpretation. The experimental work in Ref. [12] has been analyzed according to the "proper scaling law" from Ref. [10], where the temperature dependence of the anomalous Hall resistivity has been embedded into intrinsic contribution. Analyzing the same data with our scaling law with temperature-independent parameters provides deeper understanding and leads to different conclusions about the weight of intrinsic contribution at different temperatures in Ni, from which we confirm [13] that the proper scaling law [10] is no longer appropriate.

Since the fitting parameters are temperature/thickness independent, we can extract all fitting parameters using few data points. In Fig. 5 we plot the fitting of thickness and temperature dependence of the anomalous Hall resistivity for $\mathrm{Co}, \mathrm{Ni}$, and $\mathrm{Fe}$ with the fitting parameters extracted from part of the experimental data.

In our theory we did not include the cross impurity scattering, in which an electron skew scatters coherently off two correlated impurities located within a distance of the order of the Fermi wavelength [30], as the experiments conducted are in the dilute regime with scarce impurities. However, the effect of such terms would only modify the parameter $\beta_{I}$, and not the scaling law in Eq. (18).

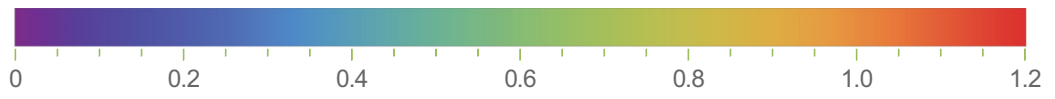

(a)

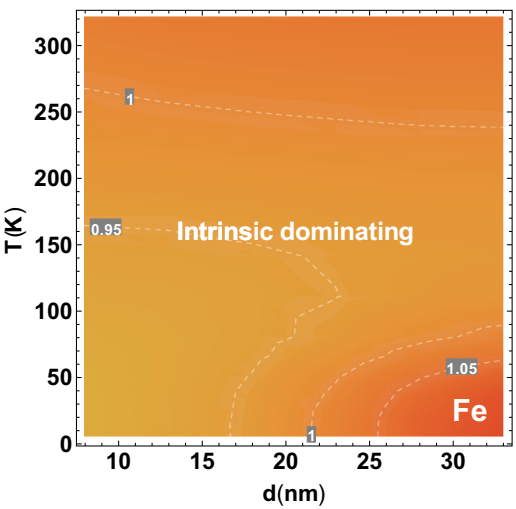

(b)

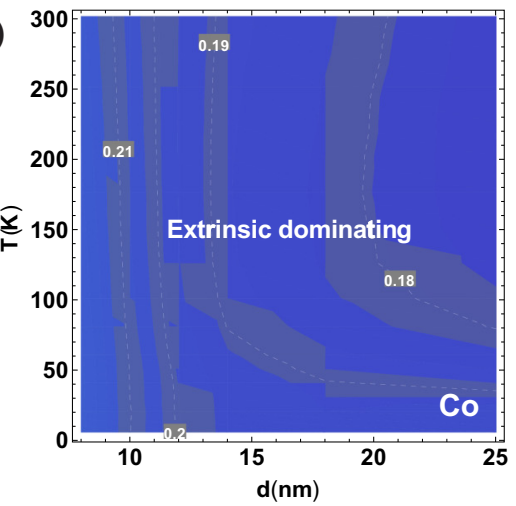

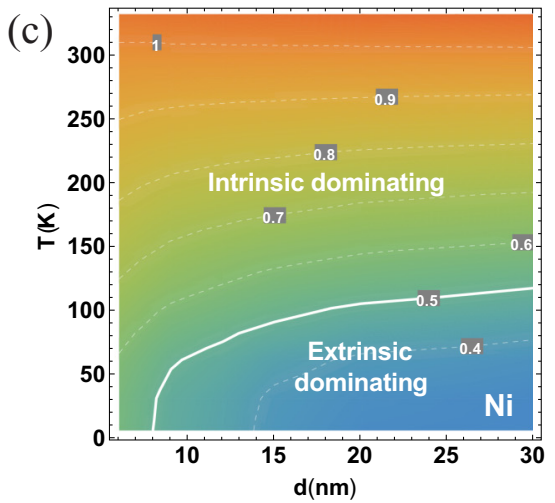

FIG. 4. The ratio of intrinsic contribution to the total AHE: $r_{\mathrm{INT}}=\rho_{\mathrm{AH}}^{\mathrm{INT}} / \rho_{\mathrm{AH}}$ as function of film thickness and temperature for Fe, Co, Ni. For Fe, the AHE is dominated by the intrinsic mechanism with $r_{\mathrm{INT}}>90 \%$. For Co, the AHE is dominated by extrinsic mechanisms with $r_{\mathrm{INT}} \sim 20 \%$. For Ni, the solid curve, corresponding to $r_{\mathrm{INT}}=50 \%$, separates the intrinsic dominating region $\left(r_{\mathrm{INT}}>50 \%\right)$ from the extrinsic dominating region $\left(r_{\mathrm{INT}}<50 \%\right)$. 
(a)

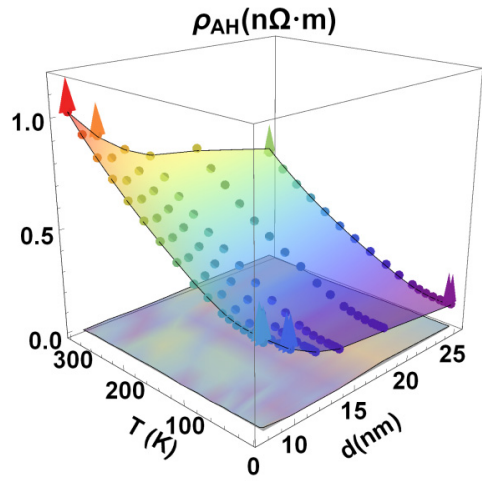

(b)

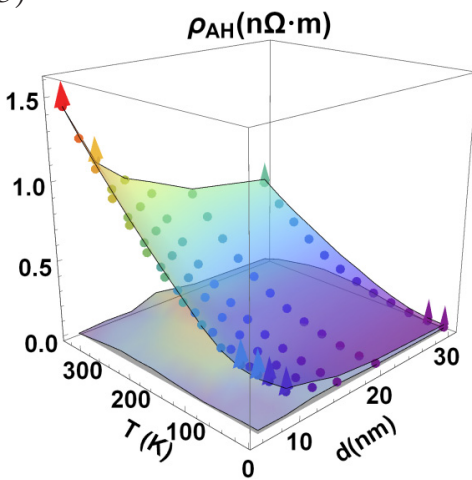

(c)

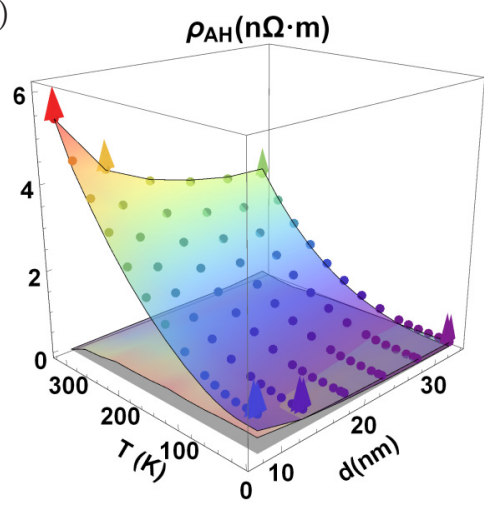

FIG. 5. The fitting of thickness and temperature dependence of the anomalous Hall resistivity for $\mathrm{Co}, \mathrm{Ni}$, and $\mathrm{Fe}$ in (a), (b), and (c), respectively, by using part of the experimental data. Points used for fitting are indicated by triangles.

\section{DISCUSSION}

Previous scaling laws $[10,13]$ are not only complex but also contingent to fitting parameters that are depending simultaneously on temperature and thickness. In contrast, our scaling law (18) with a much reduced parameter space provides an excellent agreement with the experimental data. More importantly, the fitting parameters $\alpha_{\mathrm{I}, \mathrm{P}}, \beta_{\mathrm{I}}$, and $\gamma$ are independent of film thickness or temperature. Therefore, Eq. (18) is simple and carrying a clear physical picture, i.e., separating the surface scattering from the bulk ones (impurity and phonon scattering), and is sufficient to understand both the longitudinal and transverse transport behavior in ferromagnetic thin films. With such a highly satisfied fitting to the experimental data using Eq. (18), we are able to provide an accurate estimation on the weight of each mechanism.

In conclusion, by incorporating the scatterings from surface roughness in a ferromagnetic thin film, we found that the anomalous Hall resistivity scales with the longitudinal resistivity as $\rho_{\mathrm{AH}}=\alpha_{\mathrm{I}} \rho_{\mathrm{I}} \rho+\beta_{\mathrm{I}} \rho_{\mathrm{I}}+\alpha_{\mathrm{P}} \rho_{\mathrm{P}} \rho+\gamma \rho^{2}$. In Fe, Co, and $\mathrm{Ni}$ thin films, this simple yet elegant relation agrees excellently with the experimental data. We conclude that the intrinsic (extrinsic) mechanism dominates in $\mathrm{Fe}(\mathrm{Co})$. But, in $\mathrm{Ni}$, the relative importance of the intrinsic and extrinsic mechanisms depends on temperature and film thickness.

\section{ACKNOWLEDGMENTS}

J.X. thanks X. Jin of Fudan University and A. Granovsky of Moscow State University for valuable discussions. This work was supported by the National Key Research and Development Program of China under Grants No. 2017YFA0303300 and No. 2016YFA0300702, the National Natural Science Foundation of China under Grants No. 11474065, No. 11734004, No. 61774017, and No. 21421003.

\section{APPENDIX A: CALCULATION OF SIDE-JUMP CONDUCTIVITY}

From Eq. (11)

$$
\mathbf{J}=\frac{e}{v} \sum_{s, \mathbf{q}, n}\left(f_{\mathbf{q}, n, s}^{(0)}+f_{\mathbf{q}, n, s}^{(1)}+f_{\mathbf{q}, n, s}^{(2)}\right) \mathbf{v}_{s c},
$$

where the velocity expectation value has the form

$$
\begin{aligned}
\mathbf{v}_{s c} & =\frac{\hbar \mathbf{q}_{s}}{m}-\sum_{i=\mathrm{I}, \mathrm{P}} s \frac{\eta_{i} n_{i}}{\hbar^{3}} \delta_{s s^{\prime}} \frac{V_{i}^{2}}{d}\left(\mathbf{q}_{s} \times \hat{\mathbf{z}}\right)\left(n_{c, s}+\frac{1}{2}\right) \\
& \equiv \mathbf{v}_{n}+\sum_{i=\mathrm{I}, \mathrm{P}} \mathbf{v}_{a n, i},
\end{aligned}
$$

where $n_{c, s}=k_{F, s} d / \pi$. Without losing the generality, we present the calculations for the impurity scattering. The sidejump contribution is proportional to

$$
\mathbf{J}^{s j, \mathrm{I}}=\frac{e}{v} \sum_{s, \mathbf{q}, n} f_{\mathbf{q}, n, s}^{(1)} \mathbf{v}_{a n, \mathrm{I}}
$$

Assuming the electric field in the $\hat{\mathbf{x}}$ direction and inserting Eqs. (A2) and (12) into Eq. (A3) we obtain

$$
\begin{aligned}
J_{y}^{s j, \mathrm{I}}= & \varepsilon \frac{e}{v} \sum_{s} \sum_{n} s \frac{e \eta_{\mathrm{I}} \hbar}{m} \frac{\tau_{n}^{s}}{\tau_{i}}\left(1-\frac{J_{s d}}{2 E_{F}}\right) \\
& \times \sum_{\mathbf{q}_{s}} q_{x, s}^{2} \delta\left(E_{\mathbf{q} n s}-E_{F}\right) .
\end{aligned}
$$

The summation over $\mathbf{q}_{s}$ gives

$$
\begin{aligned}
\sum_{\mathbf{q}_{s}} q_{x, s}^{2} & =\frac{A}{4 \pi^{2}} \pi \int q_{s} d q_{s} \delta\left(E_{\mathbf{q} n s}-E_{F}\right) \\
& =A \frac{m \pi}{4 \hbar^{2} d^{2}}\left(n^{2}-n_{c, s}^{2}\right) .
\end{aligned}
$$

Using $\sigma_{\mathrm{I}}=n_{e} e^{2} \tau_{\mathrm{I}} / m$ we obtain from Eqs. (A4) and (A5)

$$
\sigma_{\mathrm{AH}}^{s j, \mathrm{I}}=J_{y}^{s j, \mathrm{I}} / \varepsilon=\alpha_{\mathrm{I}} \frac{\sigma}{\sigma_{\mathrm{I}}}, \quad \text { where } \quad \alpha_{\mathrm{I}}=\eta_{\mathrm{I}} \frac{n_{e} e^{2}}{2 \hbar} \frac{J_{\mathrm{sd}}}{E_{\mathrm{F}}} .
$$

\section{APPENDIX B: CALCULATION OF SKEW-SCATTERING CONDUCTIVITY}

The skew-scattering contribution is proportional to

$$
\mathbf{J}^{s s, \mathrm{I}}=\frac{e}{v} \sum_{s, \mathbf{q}, n} f_{\mathbf{q}, n, s}^{(2)} \mathbf{v}_{n} .
$$


Inserting Eqs. (A2) and (13) into Eq. (B1), we obtain

$$
\begin{aligned}
\mathbf{J}^{s s, \mathrm{I}} & =\frac{e \hbar}{m v} \sum_{s, \mathbf{q}, n} \mathbf{q}_{s}\left[\frac{e \hbar}{m} \tau_{n}^{s} \sum_{\mathbf{q}^{\prime} n^{\prime} s^{\prime}} \tau_{n^{\prime}}^{s^{\prime}} P_{n \mathbf{q} s}^{n^{\prime} \mathbf{q}^{\prime} s^{\prime}(2)} \boldsymbol{\varepsilon} \cdot \mathbf{q}_{s} \delta\left(E_{\mathbf{q}^{\prime} n^{\prime} s^{\prime}}-E_{F}\right)\right] \\
& =\frac{e \hbar}{m v} \sum_{s, \mathbf{q}, n} \mathbf{q}_{s}\left[\frac{e \hbar}{m} \tau_{n}^{s} s \eta_{\mathrm{I}} \sum_{\mathbf{q}^{\prime} n^{\prime} s^{\prime}} \tau_{n^{\prime}}^{s^{\prime}} \frac{\pi V_{\mathrm{I}} m n_{\mathrm{I}} n_{c}}{d \hbar^{3} v}\left(\mathbf{q}_{s} \times \mathbf{q}_{s^{\prime}}^{\prime}\right)_{z} \delta_{s^{\prime} s} \delta\left(E_{\mathbf{q} n s}-E_{\mathbf{q}^{\prime} n^{\prime} s^{\prime}}\right) \boldsymbol{\varepsilon} \cdot \mathbf{q}_{s} \delta\left(E_{\mathbf{q}^{\prime} n^{\prime} s^{\prime}}-E_{F}\right)\right] .
\end{aligned}
$$

With $\boldsymbol{\varepsilon} \| \hat{\mathbf{x}}$ we further make the summations as that for side-jump contribution and obtain

$$
\sigma_{\mathrm{AH}}^{s s, \mathrm{I}}=J_{y}^{s s, \mathrm{I}} / \varepsilon=\beta_{\mathrm{I}} \frac{\sigma^{2}}{\sigma_{\mathrm{I}}}, \quad \text { where } \quad \beta_{\mathrm{I}}=-\eta_{\mathrm{I}} \frac{\pi n_{e} m V_{\mathrm{imp}}}{2 \hbar^{2}} \frac{J_{\mathrm{sd}}}{E_{\mathrm{F}}} .
$$

[1] E. M. Pugh and N. Rostoker, Rev. Mod. Phys. 25, 151 (1953).

[2] N. Nagaosa, J. Sinova, S. Onoda, A. H. MacDonald, and N. P. Ong, Rev. Mod. Phys. 82, 1539 (2010).

[3] R. Karplus and J. M. Luttinger, Phys. Rev. 95, 1154 (1954).

[4] G. Sundaram and Q. Niu, Phys. Rev. B 59, 14915 (1999).

[5] J. Smit, Physica (Amsterdam) 21, 877 (1955).

[6] J. Smit, Physica (Amsterdam) 24, 39 (1958).

[7] L. Berger, Phys. Rev. B 2, 4559 (1970).

[8] T. Miyasato, N. Abe, T. Fujii, A. Asamitsu, S. Onoda, Y. Onose, N. Nagaosa, and Y. Tokura, Phys. Rev. Lett. 99, 086602 (2007).

[9] S. Onoda, N. Sugimoto, and N. Nagaosa, Phys. Rev. Lett. 97, 126602 (2006).

[10] Y. Tian, L. Ye, and X. Jin, Phys. Rev. Lett. 103, 087206 (2009).

[11] D. Hou, Y. Li, D. Wei, D. Tian, L. Wu, and X. Jin, J. Phys.: Condens. Matter 24, 482001 (2012).

[12] L. Ye, Y. Tian, X. Jin, and D. Xiao, Phys. Rev. B 85, 220403 (2012).

[13] D. Hou, G. Su, Y. Tian, X. Jin, S. A. Yang, and Q. Niu, Phys. Rev. Lett. 114, 217203 (2015).

[14] N. A. Sinitsyn, A. H. MacDonald, T. Jungwirth, V. K. Dugaev, and J. Sinova, Phys. Rev. B 75, 045315 (2007).

[15] S. Pavlov and Y. A. Firsov, Fiz. Tverd. Tela (Leningrad) 7, 2634 (1965) [Sov. Phys.-Solid State 7, 2131 (1966)].

[16] S. Pavlov and Y. A. Firsov, Fiz. Tverd. Tela (Leningrad) 9, 1780 (1967) [Sov. Phys.-Solid State 9, 1394 (1966)].
[17] Z. Tesanović, M. V. Jarić, and S. Maekawa, Phys. Rev. Lett. 57, 2760 (1986).

[18] N. Trivedi and N. W. Ashcroft, Phys. Rev. B 38, 12298 (1988).

[19] L. Zhou, V. L. Grigoryan, S. Maekawa, X. Wang, and J. Xiao, Phys. Rev. B 91, 045407 (2015).

[20] X. Wang, J. Xiao, A. Manchon, and S. Maekawa, arXiv: 1407.8278 .

[21] S. Takahashi and S. Maekawa, Sci. Technol. Adv. Mater. 9, 014105 (2008).

[22] J. M. Ziman, Electrons and Phonons: The Theory of Transport Phenomena in Solids (Oxford University Press, Oxford, 2001).

[23] E. Grüneisen, Ann. Phys. 408, 530 (1933).

[24] A. Bid, A. Bora, and A. K. Raychaudhuri, Phys. Rev. B 74, 035426 (2006).

[25] N. Ashcroft and N. Mermin, Solid State Physics (Saunders College, Philadelphia, 1976).

[26] F. Batallan, I. Rosenman, and C. B. Sommers, Phys. Rev. B 11, 545 (1975).

[27] H. Ehrenreich, H. R. Philipp, and D. J. Olechna, Phys. Rev. 131, 2469 (1963).

[28] A. V. Vedyaev, A. B. Granovskii, and O. A. Kotel'nikova, Kinetic Phenomena in Disordered Ferromagnetic Alloys [in Russian] (Moscow State University Press, Moscow, 1992).

[29] A. Crépieux and P. Bruno, Phys. Rev. B 64, 014416 (2001).

[30] I. A. Ado, I. A. Dmitriev, P. M. Ostrovsky, and M. Titov, Europhys. Lett. 111, 37004 (2005). 